\title{
Pratiques
}

Linguistique, littérature, didactique

\section{Récit(s), figures du sens, littérature et Institution scolaire. Quelques coups de sonde}

\section{Frédéric François}

\section{(2) OpenEdition}

\section{Journals}

Édition électronique

URL : http://journals.openedition.org/pratiques/1157

DOI : $10.4000 /$ pratiques. 1157

ISSN : 2425-2042

Éditeur

Centre de recherche sur les médiations (CREM)

\section{Édition imprimée}

Date de publication : 15 juin 2008

Pagination : 146-164

\section{Référence électronique}

Frédéric François, «Récit(s), figures du sens, littérature et Institution scolaire. Quelques coups de sonde », Pratiques [En ligne], 137-138 | 2008, mis en ligne le 15 juin 2008, consulté le 19 avril 2019 URL : http://journals.openedition.org/pratiques/1157; DOI : 10.4000/pratiques. 1157

(c) Tous droits réservés 


\section{Récit(s), figures du sens, littérature et Institution scolaire. Quelques coups de sonde}

\section{Frédéric FRANÇOIS}

Université René Descartes, Paris

\section{Quelques limites de ce texte}

Bien sûr, il y a les limites de l'auteur. D'abord dans sa relation à J.-F. Halté. Il m'est difficile de suivre tout son parcours, d'autant qu'il a sans cesse modifié son approche, en s'efforçant toujours de replacer l'analyse langagière dans le cadre d'une réflexion sur notre société, l'institution scolaire, le dialogue pédagogique et la culture.

Et puis, il me semble que les termes réunis dans le titre ne concernent pas des objets sur lesquels on peut prétendre « dire le vrai » mais seulement « essayer de s'orienter», entre perspectives hétérogènes et sans bases assurées. D'où la justification du sous-titre, qui n'est pas une forme rhétorique convenue de captatio benevolentiae.

L'auteur sait bien que ses « idées », comme celles d'un personnage de roman, ne sont qu'un point de vue, apparu de façon relativement aléatoire dans son histoire, quel que soit le degré éventuellement élevé de sa conviction... Cela dit, et l'aléatoire des conditions d'apparition et le changement des convictions de chacun ne sauraient justifier, à mon sens, le scepticisme à l'égard de ces convictions : il peut y avoir urgence polémique contre une doxa dominante ou enthousiasme dans la « découverte », même relative, d'un point de vue. Ce qui n'empêche pas que, plus tard, le point de vue qui a fait choc peut devoir être abandonné, par exemple s'il devient à son tour doxa ou s'il s'avère qu'il s'accompagnait, ce qui est sans doute inévitable, de la perte ou de l'occultation d'une autre perspective : un point de vue accentue et ne saurait tout accentuer (axiome structurel indépassable?).

Autre difficulté : je ne me suis pas confronté pour l'essentiel aux exigences effectives de la didactique du français dans l'institution scolaire. Et je ne suis pas non plus «théoricien » de la didactique du français. Il s'agit donc seulement de 
remarques «venues de l'extérieur», sans que je puisse justifier le choix forcément limité des perspectives.

J'essaye donc de présenter d'abord quelques considérations négatives, puis d'évoquer quelques aspects des relations du «narratif » et son entremêlement à d'autres figures du sens dans ce qu' on peut regrouper sous « littérature », littérature des « littérateurs » ou produite en milieu scolaire, pour conclure par quelques points sur la relation intergénérationnelle, en particulier dans notre «modernité ».

Et j'espère qu'aucun lecteur ne me reprochera de ne présenter que quelques courts textes, anonymes, d'enfants sans éclaircissements quant aux conditions de production de ces textes. Ces textes sont courts. Certes, en fonction des dimensions d'un article. Un peu aussi sans doute par position polémique : il me semble qu' on juge souvent les élèves en fonction de leur capacité à faire des textes «plus longs »... Le contraire pourrait se justifier.

\section{Quelques difficultés ou impossibilités}

La première difficulté qu'on peut évoquer ici est celle du babélisme. Supposons qu'il puisse/doive y avoir une « science des textes» (ou plus spécifiquement une «narratologie ») et que cette science des textes puisse/doive nous dire comment aider les élèves à «bien lire » les textes ainsi qu'à en produire (à l'oral ou à l'écrit, c'est une autre question). Tout d'abord, si science il y a, elle est en train de se faire et ne constitue pas un corpus achevé qui dirait le vrai. Le regard rétrospectif sur ce qui a pu fonctionner comme « doxa dominante » (qu'on pense au modèle proppien, à ceux tirés de Greimas, de Labov... ou aux efforts pour classer des genres et des types de textes) nous rend, il me semble, pour le moins " relativistes », les théories évoluant, déplaçant plutôt leurs champs qu'approfondissant un même objet.

Plus généralement, on peut douter de l'énoncé aristotélicien sans cesse répété selon lequel il n'y aurait de science que du général, énoncé qui s'accompagne d'une sorte d'imaginaire platonicien selon lequel le penseur s'élève au-delà des apparences et/ou du concret (narratif), ce qui a encore des traces quand on progresse (?) dans notre cursus scolaire des classes de lettres à l'enseignement de la philosophie. Rien ne justifie un tel essentialisme. Pourquoi le général serait-il l'essentiel au sens du fondamental de ce qui permet de penser « la nature des choses » ? Corrélativement, ne devrions-nous pas nous méfier de notre capacité (inscrite dans la « nature de la langue » ?) à considérer des noms d'abstraits comme s'ils désignaient des "idéalités réelles » : l'intelligence, la justice, l'inconscient, le désir ou le récit...?

Assurément, on peut appeler « intelligent» celui qui a résolu un problème, saisi le sens d'une allusion ou trouvé l'attitude à avoir à l'égard de celui qui est en face de lui. En revanche les liens entre l'intelligence des problèmes, celle des allusions et celle des situations ont au mieux un " air de famille ». Certes, ces « grands mots » ne peuvent être évités. Il serait impossible de dire qu'il n’y a que des intelligences, des inconsciences, des récits différents et de nommer chacun d'eux d'un nom différent. La solution intermédiaire est peut-être que ces grands 
mots obéissent à la « logique de la langue ordinaire » et que de même que les relations entre les sens d' " aimer» « le vin », " ses enfants », « Dieu » sont pour le moins tendues, de même toute utilisation d'un de ces « grands mots » sans plus de précision n'ira pas de soi : il n'y a pas là indication de genres où les espèces viendraient se ranger sagement.

Il me semble plutôt, pour proposer une «généralité négative », qu'il n’y a pas une règle universelle qui nous dirait a priori ce que doivent être les rapports entre «le commun» et « le spécifique ». Il y a bien quelque chose comme un monde partagé, comme des "existentiaux », naître, mourir, travailler, se souvenir, vieillir, être avec d'autres ou seul... Mais tout autant tension, opposition entre les modes concrets de manifestation de ces généralités. En particulier ici, dans le cas des textes, il y a du «proche » plus que du «même ». Mais surtout, on peut douter qu'il y ait une « bonne perspective » qui permettrait de rendre compte de ce qu'un texte a de commun avec d'autres et de ce qu'il a de spécifique, tant dans ce qui a permis sa production que dans les modes de réception qu' " on » peut en avoir. Assurément, il faut tenir compte de l'amont des formes de langage reçues, des capacités de mélange comme d'élaboration réflexive, qui se trouvent en tout auteur, «grand homme » célèbre ou enfant parmi d'autres. Tout comme de la relation a ce qui est admis, norme locale ou générale, ou vécu comme transgression. Ou, plus encore, du dialogue « dans » l'auteur comme dans notre réception entre le sens manifesté à travers les mots et les sens autres, ce qui relève de la "passivité créatrice " commune à ce qui se passe dans le rêve, la rêverie ou dans la machine à produire des textes, de l'utilisation de schèmes acquis ou d'un travail d'élaboration second... le rapport entre dit, indiqué dans le mouvement du texte et hors langage restant assurément lui-même du " difficile à dire ». Nous n'avons pas une métathéorie mettant en place les aspects hétérogènes de ce qui permet de comprendre (bien sûr pas d'expliquer « complètement ») la façon dont tel texte se constitue ainsi que les modalités variées de sa réception. Plus généralement, je doute qu'on puisse décomposer en éléments ultimes (psychologiques, sociaux ou autres) le processus de production. Il reste toujours, qu'il s'agisse de médecine, de cuisine ou de «textologie » une distance entre les modèles théoriques et la réalité des productions concrètes. Pas plus qu'on n'explique complètement le processus de production, on ne rend compte de celui de réception où le texte, juxtaposition de signes, est transformé en mouvements de sens, en «choses mentales », ce qui suppose le travail, largement non explicite, des arrièrefonds qui rendent cette transformation possible, qu'il s'agisse des arrière-fonds communs ou supposés communs ou des modes plus spécifiques de réception (sans que nous ayons à chaque fois un indice de ce degré de spécificité : comme on le sait, l'erreur ici est commune). Par ailleurs cette réception peut rester à l'état implicite ou se manifester sous les formes de compréhension responsive explicite que sont par exemple la question ou le commentaire. Nous sommes dans un espace de dialogue, fait de différences entre ce que nous pouvons produire et ce que nous pouvons recevoir, comme entre notre pratique (d'auteurs ou de lecteurs) et la théorie que nous en faisons. Même s'il y a des points d'accord collectif, cela ne constitue aucune assurance de «vérité ». Sommes-nous capables de faire la théorie des modes de retentissement d'un texte, des effets d'un style? En tout cas, on est dans le difficile à penser, à dire. Ici on ne peut proposer des concepts aux limites strictes, plutôt des notions qui permettent peut-être d'éclairer, 
sûrement pas de fixer des frontières assurées et qui sont toujours susceptibles de modifications tant dans la rencontre de nouveaux objets que d'autres modes de réception (des autres ou de soi). On peut (peut-être) parler du roman en général, mais cela ne nous permet pas de séparer « roman » et " non-roman » encore moins de dire quoi que ce soit sur les romans qui n'existent pas encore, ni de nous donner une «loi d'engendrement». Tout comme c'est une banalité de dire qu'Ulysse ou la Recherche du Temps perdu ont d'autres « organisateurs dominants » et nous donnent d'autres figures du roman que ceux qu'on pourrait tirer d'une définition abstraite du roman.

Et il reste, il me semble, de la vérité dans le Contre Sainte-Beuve : ce ne sont pas des savoirs sur l'auteur qui nous diront quelque chose sur son œuvre et les échos qu'elle peut avoir.

Pas plus qu'il n'y a (me semble-t-il) un métadiscours qui nous permettrait d'épuiser la diversité des perspectives qui peuvent être prises, aussi bien sur « le récit » que sur tel récit.

On demande donc l'indulgence en proposant quelques « points de vue généraux » sans pouvoir justifier absolument ce qui est avancé, ni, encore moins, l'élimination (volontaire ou non) de telle ou telle perspective. D'autant qu'après avoir proclamé une méfiance à l'égard des assertions essentialistes, on ne se prive pas d'un degré redoutable de généralité, comme l'illustre la première proposition qui va suivre.

\section{Quelques propositions}

\subsection{Le langage et son arrière-fond}

Ce titre pour rappeler qu' on parle ici des textes (sans distinguer « textes » et « discours ») et des différentes relations qu'ils permettent entre leurs « interlocuteurs » et non de l'objet abstrait, fictif, qu' on appelle langue. On s'accorde pour reconnaître qu'à travers l'exercice du langage (et en particulier par le récit) peuvent nous être donnés différents types de significations et/ou de savoirs que la seule relation perceptive ou l'acte moteur ne peuvent nous donner. Mais il faut ajouter en même temps que si un énoncé peut « faire sens » pour nous, c'est qu'il est en relation à autre chose que lui et d'abord à d'autres figures du sens, en particulier, celles que nous partageons partiellement avec les animaux ou les enfants avant que ceux-ci n'aient recours au langage. Tout d'abord, les corps vivantspensants sont en rapport avec des «totalités signifiantes » : nous voyons quelqu'un qui s'approche de nous, non une succession de sensations reliées ensuite par la «pensée ». Corrélativement, le « sens » n'est pas d'abord une relation signifiant-signifié, mais une relation de prise dans un ensemble, qui n'est pas portée par les seules perception et cognition, est plutôt l'effet global d'un « mode de retentissement ». Ces modes de retentissement sont, si on veut, "affectifs », mais ont peu à voir avec la classification traditionnelle des émotions, ce que rappelle l'évocation de termes comme ceux du familier, de l'étrange, du fascinant ou du « à fuir » ou encore du neutre (Barthes). Chacun de ces termes n'est pas exclusif des autres, tout comme il va se modaliser, acquérir une spécificité, assuré- 
ment difficile à dire, selon les cas. Tout cela, en particulier entre un adulte et un enfant, comme entre soi et soi, comporte de la communauté et de la différence : plutôt que de parler d'un «monde commun », on pourrait s'interroger sur le " commun dans le monde ». Mais sans cesse nous prenons comme commun ce qui ne l'est pas et inversement nous trouvons de l'analogue là où un autre n'en voit pas.

Je rappelle une citation bien connue de Volochinov, qui vise à ouvrir la signification de la notion de « signe» (p. 50) : «Qu'est-ce qui constitue le matériau sémiotique du psychisme? Tout geste ou processus de l'organisme : la respiration, la circulation du sang, les mouvements du corps, l'articulation, le discours intérieur, la mimique, la réaction aux stimuli extérieurs (par exemple la lumière) bref, tout ce qui s'accomplit dans l'organisme peut devenir matériau pour l'expression de l'activité psychique, étant donné que tout peut acquérir une valeur sémiotique, tout peut devenir expressif ». Ce passage est évidemment rapide, laisse sans explicitation, entre autres, la question de « expressif pour l'autre » et « expressif pour soi ». Reste que Volochinov lie ainsi deux dimensions : d'un côté c'est le corps en lui-même qui est source de signes. De l'autre, il n'y a de signe que par le lien aux autres (ou à soi comme autre). "Signes » est-il le bon mot ? On peut en douter en ceci que le renvoi d'un élément à un ensemble (pas forcément identique) le «met en sens » sans qu'il y ait signe à proprement parler. Ce qui « fait sens » c'est l'ensemble de ce qui est « perçu en tant que », en même temps ressenti et source de réaction, «sens-force». La base de tout cela c'est le corps de chacun comme centre fragile du monde où les différentes sources de sens : le perçu-ressenti, les modes de réaction, le présenté par les autres (leur corps ou leur langage), le proche, le lointain et l'absent sont en relation. Tout cela dans une communauté-différence, un peu comme les sens à distance, vue et audition surtout, d'une part régulent les mouvements du corps, en même temps que chacun de ces sens peut fonctionner selon son ordre propre : un rythme peut être partagé entre modalités sensorielles. Pas une couleur ni une mélodie. C'est le sort de tout ce qui permet ou non de s'orienter, d' "être au monde » avant qu'il soit question de «valoir pour». En même temps, ce corps vécu est aussi en quelque façon le corps «objet » et en particulier « objet de science », question impossible à traiter ici. On notera que la mise en science n'est qu'un cas particulier de l' « inversion sémiotique » qui fait que le monde vécu peut être en quelque sorte pris dans le monde des significations dites ou, plus généralement, représentées.

En tout cas, lorsqu' on perçoit un texte comme texte, il s'agit bien de le percevoir comme mouvement plus ou moins complexe et non comme succession de segments (ce qui serait « ne pas le comprendre»). Si on prend l'exemple du récit de rêve suivant écrit par un jeune enfant :

Une nuit j'ai rêvé d'un monsieur qui venai du plancher. Il venai droit vers ma chambre

il ouvrai le porte il voule me couper ma tête on entendit du bruit puis il m'a couper la tête.

$$
\text { Jean Paul }
$$

chacun de nous (?) perçoit une unité, un mouvement composés de différents ensembles : par exemple, les ensembles périphériques de l'annonce et de la signature et la continuité des épisodes d'un même récit de rêve. Il y a bien « narrativi- 
té » si l'on entend par là une succession discursive en rapport avec une succession qui a eu lieu. Il y a, plus précisément, unité d'une « scène », les « scènes » constituant sans doute des unités de sens antérieures à l'entrée dans le langage. Mais ici le langage ajoute ses significations propres qui peuvent être en même temps difficultés à dire le rêve. D’un monsieur qui venai du plancher reste opaque. Mais le lecteur peut aussi accepter cette opacité. De même qu'on ne peut pas savoir le pourquoi de l'interruption de la séquence de l'action du «monsieur » par : on entendit du bruit. Mais il me semble que cette prise en compte du sensible s'intègre ici dans l'étrangeté du rêve rapporté. Puis, que le rêve soit une totalité se manifeste par la rétroaction de la fin sur le début : ce n'est que quand on rapporte un rêve que l'on peut dire il m'a coupé la tête. Sans parler de l'effet de choc qui vient de ce que rien de plus ne soit dit.

Toujours est-il qu'un second moment consistera à s'interroger sur les relations entre ces différentes sémioses, ici le dire et le rêver, en notant que même s'il est vrai qu' on peut raconter un rêve qui n'a pas eu lieu, la chute (pour moi) « sonne vrai », n'a sans doute pas pu être inventée. En même temps que personne ne saura jamais la relation exacte du récit au rêve. Mais surtout, et c'est là que la division en genres ne nous aide pas, ce qui importe ce n'est pas l'appartenance ce texte au genre « récit», mais la question de la façon particulière dont il est récit. Ainsi dans le lien entre l'étrangeté montrée et non dite du rêve et le caractère minimal du récit, l'absence d'explicitations, de cadre, de modalisations, tout ce qui contribue négativement à « faire effet». Mais cela c'est ma façon de le lire. Non une caractéristique du «texte en lui-même ». Ou, plutôt, ce texte est entre nous, comme nos vies sont tissées des communautés-différences entre nous et les autres et comme nous sommes « sujets » en entendant par là l'ensemble des façons d'être hétérogènes constituées par un corps ressentant, réagissant, alternativement seul et proche des autres, présent à ce qui l'entoure comme aux différentes modalités de l'absent et d'abord à celles qui passent par le dit ou l'écrit.

J.-F. Halté évoquait peu sa vie personnelle dans ses textes. C'est bien cependant la proximité-distance entre vie et textes qui se manifeste dans son article «Apprendre autrement à l'école ». L'article commence par le récit d'une anecdote : il va à la ferme avec son petit garçon de trois ans, qui hésite à passer la barrière d'entrée parce qu'il y a « un loup».

D'où le dialogue « explicatif» :

- Mais non, gros bêta, c'est un chien, pas un loup.

- Pourquoi?

- Tu sais bien qu'il n'y a pas de loup dans les fermes et les villages.

D'où la remarque toujours adéquate, même si pas toujours respectée dans la vie scolaire : expliquer n'est pas une fin en soi, mais une façon de faire qui ne se développe que lorsqu'il y a problème. Puis l'explication passe à une autre question :

- Pourquoi il a les yeux brillants?

qui renvoie à l'ensemble difficilement assignable des loups de contes, en particulier celui de la chèvre de Monsieur Seguin et des jeux de l'enfant qui se rejoue la saynète célèbre pour son propre compte, avec la fascination de la nuit où quelque chose, un regard brille, saynète « où il joue de plus en plus le rôle du loup ». 
On voit ici le passage de la première opportunité : celle de la rencontre et de la seconde : l'arrière-fond hétérogène qui constitue l'« expérience pathique »du sujet. Cet arrière-fond est plus ou moins présent au père. Mais surtout, celui-ci a répondu en parlant et non, par exemple, en donnant un coup de pied au chien ou en tirant l'enfant par le bras. Si l'on veut, le père a introduit un autre espace que celui de l'événement, ce qu'on peut appeler un « espace de suspension ». Mais on voudrait revenir, avant de caractériser cet espace, sur deux aspects plus généraux du «faire sens » : la relation des «fermés » et des « ouverts », la relation obligée au temps.

\subsection{Le sens comme relation entre des fermés et des ouverts, atmosphères et associations}

Le mouvement comme ensemble peut à son tour être pris (ou plutôt ne peut pas ne pas être pris) dans d'autres ensembles : voir venir vers moi quelqu'un entrera dans le champ d'autres perceptions analogues, entre l'étonnement, le plaisir, la peur... Avant tout langage, 1' infans réagira différemment à différentes modalités du geste. L'évolution de la culture intériorisée sous forme de nature aboutira à la multiplication des modes de perception, des « ouverts » dans lesquels un même mouvement pourra se placer. Une des premières figures du sens doit bien se présenter en quelque sorte comme un « il y a» ou un « il se passe», relativement assignables, mais toujours corrélatifs d' «arrière-fonds ». Sans qu'on sache très bien comment caractériser en général ces arrière-fonds : sans doute, pas seulement un fond comme celui de la perception visuelle, quelque chose comme un mixte de présence et d'absence, d'explicite ou non avec toute l'hétérogénéité de ce qu'on appelle «monde », chacun des aspects de cet arrière-fond se modalisant ou se mêlant à d'autres selon la spécificité de la situation. Et puis, entre ce qui est strictement figure ou fond, vient se greffer la circulation d'autres éléments, l'ensemble de l'associatif, en particulier du « un peu comme » dont la figure est ellemême plus ou moins assignable. Moins dans la perception, le rêve ou la rêverie, davantage dans les mouvements textuels. Ce peut être ce qui nous fait passer d'un « thème » à un autre. Cela peut prendre, dans les textes, la forme de la parataxe en général ou celle plus spécifique de la liste, activité sérieuse ou exemple du pur plaisir à manier le langage. Par opposition au langage de la pratique, on pourrait dire que le pôle parataxique serait le représentant même de l'espace de suspension, de l'inspiration ou de la création passive, selon des modes variés chez chacun d'entre nous. Mais les textes, la musique ou le devenir des images nous mettent en relation avec des régimes de sens-force différents de ceux qui constituent nos lignes intimes de plus grande pente. On ne peut opposer ici simplement ce qui serait « de nous » et « pas de nous ». Il y a l'ensemble de tout ce qui est « de l'autre en nous».

Je donne ici un exemple qui a déjà été développé plus longuement ailleurs. On a demandé à des enfants de diverses classes (ici CE 2) de raconter successivement une histoire horrible puis un cauchemar.

Laetitia, Histoire horrible

Il y a longtemps dans un pays, un monstre a écrasé les hommes, les femmes, les enfants, il écrasait tout et on voyait les boyaux éclater, le sang coulait. 


\section{Cauchemar}

Une nuit, j'ai rêvé d'un dragon qui mangeait tout le monde et coupait la tête aux bébés. J'étais la dernière à me faire brûler la tête. Il cassait toutes les maisons puis écrabouillait les hommes, les enfants et les bébés.

Dans les deux cas, la logique de l'accumulation est en affinité avec la logique de l'horreur. Une grosse différence : dans le rêve, $j e$ «se fait brûler la tête ». Avec le parallèle que cela comporte avec le récit de rêve précédemment rapporté. Avec aussi la modification de la dramatisation liée au fait que le texte ne s'arrête pas là, mais aussi au fait que la destruction de «moi » est prise dans l'accumulation, sans qu'on puisse rendre compte du passage de la première série, normée : écraser hommes, femmes, enfants à écrabouiller hommes, enfants et bébés qui l'est moins. On ne peut ici, pas plus que dans le rêve précédemment rapporté, savoir ce qu'a été le rêve effectif. Mais l'effet de ce qui est rapporté se mêle à la « signification seconde » portée justement par la spécificité de l'organisation textuelle. Sans que des récits d'horreur doivent nécessairement prendre une telle forme. Bien sûr, il y a une «naturalité » ou des « affinités » qui font qu'on raconte autrement, pour prendre des stéréotypes, «la mort d'un être cher» et « une belle journée à la campagne ». Mais ces affinités peuvent être transgressées sur le mode du petit tailleur qui proclame « sept d'un coup ». Ce qui fait que si on considère cinquante textes portant même sur un thème qui induit le retour à du commun comme la «belle journée à la campagne ", il n'est pas sûr que les ressemblances l'emportent sur les différences. La relation thème-atmosphères-associations peut être l'objet d'une prise de conscience non d'une pédagogie sous forme de règles.

\subsection{Les orientations temporelles}

Un peu de la même façon, il n'y a pas de texte sans temps. Mais cela ne nous indique pas à quels régimes de temporalité nous allons avoir à faire.

Ici aussi de quoi partir? De la présence en nous du passé évoqué et de l'avenir envisagé ? De la façon dont du passé non représenté éclaire notre présent, sous forme de familier ou d'étrange ou de bien d'autres modes de retentissement, de même que nous sommes déjà en avant de nous sans y porter attention?

Mais il n'y a pas un temps «premier » ou « vrai». Un texte peut privilégier le temps biologique, de l'alternance entre le temps du besoin et celui du repos, de la veille et du sommeil comme de la rythmicité du corps, du cœur, de la marche... Ou celui plus long de la naissance, des âges de la vie et de la mort.

Ou encore le «temps pathique » du regret, de l'attente, de l'enthousiasme. Ou encore celui du conflit entre le temps de nous et celui de l'autre, si manifeste entre l'enfant qui désire et l'adulte qui répond «plus tard » ou dans l'incrédulité de l'enfant à qui on dit que les parents ont été enfants, que lui-même sera parent ou que lui aussi mourra.

On peut se placer ou se trouver placé dans le temps lent de l'histoire universelle, voire du cosmos ou dans le temps scandé par les événements, et leurs modes de retentissement.

Ou encore la temporalité peut s'organiser entre le temps homogène des horlo- 
ges et le temps hétérogène de la vie de chacun, scandé lui-même dans notre société et pas dans d'autres par le temps des horaires et des programmes.

Tout comme l'organisateur dominant peut être la différence entre le cours du temps auquel nous ne pouvons rien et les modifications incessantes de nos souvenirs et de nos projets.

En tout cas, « du temps » est constitutif de toutes nos façons d'être : il n'y a pas de corps, d'objets, de sentiments, de pensées qui ne deviennent pas. Et tout cela est dans différentes relations à la possibilité d'être dit explicitement ou indiqué.

On peut se concentrer ici, en gardant les modes différents qui viennent d'être évoqués comme arrière-fond, sur la différence entre le temps de ce qui a été, celui de la forme narrative et celui de la lecture. Mais de même qu'on a évoqué les relations du sens langagier et du sens premier du rapport au monde, de même on peut se demander si les temporalités du langage narratif ne prennent pas sens que par leur affinité-opposition au temps déjà là. En particulier à la prototemporalité, celle à laquelle fait allusion Winnicott lorsqu'il parle des « événements complets » que peut vivre l'infans lorsque ceux qui s'occupent de lui ne sont pas trop pressés et lui donnent ainsi le sentiment du temps : "Comprenez-vous qu'on ne peut prendre du plaisir au milieu des choses (ou les supporter si elles sont mauvaises) que si le sentiment que les choses commencent et finissent s'établit fermement?».

\subsection{Espaces-temps de suspension, langage et significations secondes}

On peut proposer qu'une des caractéristiques de la temporalité humaine est l'opposition entre le temps de l'action ou de l'affect-choc et celui de la « suspension ». Mais combien d'espèces d'espaces de suspension : espace de jeu, de rêverie, espace de célébration, espace de vide, d'ennui, de rêve aussi... ? En tout cas, la capacité à «être ailleurs » est donnée à l'homme. Ce n'est pas une pure fabrication culturelle. L'enfant rêve, joue, fait semblant, regarde autour de lui « pour le plaisir ». En cela, le langage multiplie notre capacité à « être ailleurs » (même si, c'est une autre question, le supposé « langage intérieur » est plutôt un mixte de langage et de sens corporel). Et c'est bien l'ouverture d'un tel espace de suspension qui se produit dans le dialogue du père et de l'enfant évoqué plus haut.

L'inventaire des « espaces de suspension » est sans doute impossible, tout comme celui des articulations entre les « fermés » et les « ouverts » du texte ou de l'œuvre en général.

En tout cas, dans les espaces de suspension peut se présenter tout ce qui n'est pas « possible dans le réel». Aussi bien le fictif, le jeu que les énoncés généraux (par exemple sur les loups). Ainsi, il n'y a pas une nature simple de la fiction. Elle peut être pur plaisir d'être " ailleurs » ou nous mettre en face de ce qui ne peut nous être donné, que nous préférions ne pas le voir ou qu'il soit impossible à percevoir dans la vie telle qu'elle est, par exemple lorsque le roman juxtapose, rend co-présentes les façons de saisir le monde de plusieurs participants à un même événement, qui ne nous sont jamais données en même temps dans la réalité quotidienne. Tout comme, dans l'espace imaginaire, peut être transgressée la distinction des sexes ou des âges. Tout comme l'horrible, l'interdit ou le honteux 
peuvent y être sans difficulté objet de réprobation et de jouissance. En même temps qu'à travers les œuvres il y aura inversion sémiotique, lorsque ce n'est plus le monde qui nous entoure qui éclaire le sens du dit, mais quand au contraire c'est à partir du dit que se manifestent des mondes, dans lesquels, éventuellement, nous vivons plus intensément que dans celui-ci. Aussi bien le court récit de rêve que l'échange au sujet des loups ne nous donnent pas la vérité du rêve ou de ce que c'est que le loup pour l'enfant. Plutôt la possibilité pour nous d'en avoir des "équivalents énigmatiques». Les textes des enfants nous fascinent et nous inquiètent justement parce que les enfants ne sont pas, comme le sont souvent les adultes, dans un monde stabilisé où on est censé « bien savoir » la relation entre mondes vécus et mondes dits.

Toujours est-il que ce qui apparaît dans les espaces de suspension soit reste à part, soit revient en retour sur notre perception du présent. Ainsi nos autres lointains et non seulement nos proches deviennent constitutifs de nous. Et, parmi ces autres, l'homme préhistorique, le Christ, mes parents morts, Rastignac, les fées sont tous des figures qui obéissent à d'autres façons d'exister que nos interlocuteurs actuels, sans qu'on puisse fixer avec certitude quelles sont les ressemblances et les différences des façons dont les uns et les autres agissent en nous, avec des forces homologues ou différentes. En tout cas, ces autres lointains sont constitutifs de nous, même s'il est difficile de préciser sous quelle modalité.

\subsection{Texte et hétérogénéité}

Que les mondes perçus par moi, par tel autre ou par les autres en général ne puissent être strictement équivalents peut ici apparaître comme un des aspects centraux de la non-coïncidence des « interlocuteurs » dans le dialogue large de la culture. En particulier, on y a insisté, dans la constitution des « entours », des façons de «considérer en tant que » (que ce soit sur le mode du ressenti, celui de l'associatif ou celui du commentaire explicite). Ce qui n'exclut pas l'autre aspect: « quelque chose passe » entre nous, aussi bien sur le mode de la reprise, de la traduction que de l'effet non-traductible.

A cette hétérogénéité du circuit même du sens, s'ajoute l'hétérogénéité du texte même.

En ce qui concerne le récit, caractérisé banalement par sa relation supposée bi-univoque entre succession des événements et succession des énoncés au temps dit ou manifesté, on peut opposer (au minimum) deux dimensions. Tout d'abord, la rupture de ce parallélisme : effets d'annonce, de retours en arrière, d'ellipses ou de changement de tempo. Et puis, plus généralement toutes les irruptions d'autres temporalités : fixation descriptive, primat de l'ordre associatif, parenthèses-généralités, paroles rapportées, réflexions critiques... Un « récit » n'est pas forcément d'abord ou essentiellement « récit». D'autant que l'allure du texte, son style peuvent agir en nous bien plus que ce que le texte comporte de sens traductible.

Si l'on s'interroge sur ce que peut le langage (et sur ce qu'il ne peut pas) par rapport à ce qui est possible ou impossible quand on reproduit mimétiquement l'action ou qu' on la filme, il apparaît d' une part que celui qui raconte ne peut jamais donner « la chose même ». Puis qu'il reprend, qu'il le veuille ou non, une 
part de la masse de sens potentiel accumulée dans les mots et la syntaxe. Ce qui est un facteur évident d'ouverture par rapport au seul champ corrélatif de la présentation de l'événement. Mais aussi d'ambiguïté, en particulier en fonction de l'accentuation différente qui est le fait de chacun dans l'actualisation des éléments de ce potentiel. On peut insister ici sur l'hétérogénéité comme figure centrale du livre. Ainsi, même si on peut dire que la Bible est plus une bibliothèque qu'un livre, reste que son lecteur ne peut qu'être dépassé par la multitude des figures du sens auxquelles il se heurte, effet multiplié par la distance historique et l'étrangeté.

On peut, en particulier en suivant Kundera, noter que, quelle que soit la diversité des romans, ils se caractérisent tous, plus ou moins, par l'impossibilité de les réduire à être des récits au sens linéaire. Ce qui y importe, c'est le changement de fil. L'exemple typique étant le Tristram Shandy de Sterne avec ses interruptions perpétuelles. Mais déjà Kundera rappelle que dans l'Histoire de Tom Jones de Fielding, c'est l'hétérogénéité discursive qui importe, et tout d'abord celle des titres des premiers chapitres de chaque partie qui sont des commentaires et des commentaires ironiques. Ainsi lorsqu'on rencontre le titre du chapitre 5 de la partie I : «Qui traite de quelques affaires ordinaires, ainsi que d'une remarque fort peu ordinaire à leur sujet», comme le livre III « contenant peu de choses sinon rien » ou le livre IV «contenant cinq pages de papier».

Kundera rappelle que Fielding nomme le genre de son texte : « écrit prosaï-comi-épique ».

Certes, le cours du roman ne suit pas la même voie que le cours de notre présence à nous-mêmes. Mais ces deux successions présentent un même caractère : être composées de réalités hétérogènes (sauf moments de monoïdéisme qui nous arrivent aussi) impossibles à fixer dans un « discours couvrant ».

On pourrait dire que ceci nous écarte des textes d'enfants. Encore que si on donne aux enfants la possibilité de produire des textes longs, il devient de plus en plus probable que ces textes vont multiplier les façons de faire sens qui les constituent. Et parmi les organisateurs homogènes-hétérogènes des textes d'enfants (ou d'autres), on peut revenir sur la spécificité de la liste comme genre étrange, comme mise ensemble dans un espace de suspension discursif ce qui n'était pas destiné à se réunir. (Mais, après tout, même un texte qui se veut "réaliste » met ensemble des réalités qu'on ne peut réunir dans la pratique).

Ainsi dans 1'article de M.-C. Penloup « Journaux intimes de jeunes enfants. Enjeux didactiques d'une analyse de l'écriture de soi hors école» (p. 70), je relève l'exemple suivant tiré du journal de Karine (CE1) qui constitue le début de ce journal :

Il ne faut pas être timide quand on parle au téléphone.

Il ne faut jamais se dire qu'on ne va pas y arriver.

Pour être aimer il d'abord aimer

Il ne faut être timide en aucune circonstance.

Ce texte frappe d'abord par l'aspect inattendu de cette déclaration de principe au début d'un journal. Ensuite par l'aspect non modalisé de la liste. Puis, parce que son origine reste obscure : quelle place entre le discours de l'autre et le discours de soi ? Le premier précepte est, peut-être, plus adressé de soi à soi ? Les 
trois autres, avec en particulier la clôture par « en aucune circonstance »semblent davantage venir d'ailleurs. Mais chaque fois qu' on se donne à soi-même conseils ou principes, l'identification de qui parle à travers nous n'est-elle pas un peu aussi obscure ? De toute façon l'impossibilité de décider fait partie du « charme » du texte, ce qui est sans doute plus particulièrement une des caractéristiques de la liste. Mais le « charme» vient aussi du fait qu'un journal comporte la possibilité de parler de son avenir. Si l'on veut qu'on ne peut pas déduire les " genres effectifs" des « genres annoncés».

Ce qui me frappe dans les récits qu'on a transcrits c'est qu'ils ne pourraient guère être dits autrement : c'est, peut-être, ici qu'on pourrait introduire la notion de « symbole » : une signification liée à l'adhérence à une forme (ici au texte, ailleurs à une image ou une mélodie) dans la mesure où il ne peut être traduit, mais où sa forme est là, qui transcende l'opposition général - particulier et nous donne une figure ici de la mort, de l'horrible ou ailleurs de quelque façon d'être que ce soit. Ou encore, cette prégnance de la façon de dire, cette irréductibilité des significations dessinées par le mouvement du texte aux significations dites manifeste des façons d'être et de sentir non réductibles à un concept, ou, si l'on veut, ce qu'il peut y avoir de «poétique» dans les textes les plus banals (et les plus brefs).

\subsection{L'espace fragile du « littéraire »}

Ces remarques pourraient servir à essayer de cerner l'espace du « littéraire». En notant d'abord que cet espace est instable, si on se le représente comme équilibre fragile entre deux pôles du « sens-force ». D'un côté, les symboles sociaux tels que la tradition, l'état, la religion, l'institution scolaire, les media les transmettent. De l'autre, les symboles individuels, intimes tels que les accidents de la vie font que chacun a ses propres points d'ancrage. Le domaine des symboles littéraires serait alors celui où les symboles des autres (de certains autres) nous sont proposés sans contrainte (sauf quand ils deviennent justement matière à examens ou concours). Nous les partageons plus ou moins, selon des régimes différents. Ainsi ils peuvent en nous devenir prégnants ou rester à l'état de libre jeu, de « distraction ».

On peut se demander si, au moins dans un des sens du mot, l'espace de la littérature ne consiste pas à retrouver à travers la médiation des mots l'espace des relations sensibles - ressenties, sans prétendre avoir accès à un savoir, une explication. Ce qui serait possible parce que tout texte a une signification poétique, au sens de liée à la spécificité de la forme, du mouvement comme non traductible, la poéticité première du libre jeu, de la suspension permettant, parfois, la poéticité seconde du «manifester en tant que ».

Cela dit, nous n'avons pas par-devers nous une définition préalable de ce qui pourrait s'appeler « réalité ». Certes, il y a la présence sensible, sans doute caractéristique générale de la «littérature », mais à travers ce sensible, le « réel», estce l'objet stable, le commun ? Est-ce le cours du temps ? Est-ce le mouvement intime en nous? Est-ce la différence entre ce que je perçois et ce que perçoit l'autre ? Ou le renvoi au (supposé) secret caché du monde ?... Bien évidemment, il n'y a pas ici de réponse univoque, valable pour toutes les œuvres de toutes les 
cultures, surtout si on n'oublie pas que la diversité des textes est plus que redoublée par la variété des modes de réception. Par exemple, tout texte peut se présenter comme non modalisé, sur le mode du «c'est ainsi », mais sera plutôt reçu comme : c'est ce que dit tel « sujet» assignable ou, comme ici, non assignable : « un enfant».

On vient de le dire, on propose que ce qui oppose ici « la littérature » à « la science » ou à « la religion », c'est que le texte littéraire vise à nous faire participer à une façon de faire sens, non à éliminer les autres façons de faire sens. Mais en même temps, on ne peut séparer le « littéraire » du reste de la vie. Ce que nous dit Bakhtine au début d'Esthétique et théorie du roman, quand, partant de la séparation des trois domaines de la pratique, de la science et de l'esthétique (je laisse de côté ici le rapport de Bakhtine à la religion), il note (p. 40) que le domaine culturel «n'a aucun territoire, il est entièrement situé sur des frontières qui passent partout, traversant chacun de ses aspects ». Il précise que : «Tout acte culturel vit, en substance, sur des frontières; de là son sérieux et son importance; attiré hors de ses frontières, il perd pied, devient vide, arrogant, dégénère et meurt ». Il a une " autonomie participante ». Ce qui nous écarte de la façon dont Kant voulait séparer absolument les trois domaines de la science, du jugement moral et du jugement esthétique. Bakhtine ajoute (p. 41) : « Ainsi l'acte cognitif trouve une réalité déjà élaborée dans les concepts (je dirais les notions et les pratiques) de la pensée préscientifique, mais surtout déjà appréciée et réglementée par l'acte éthique, quotidien, social, politique ; il la trouve affirmée avec ferveur. Enfin l'acte cognitif découle de la représentation de l'objet esthétiquement ordonné, de la vision de l'objet. » Ce qu'il complète (p. 44) : « l'activité esthétique ne crée pas une réalité entièrement nouvelle. A la différence de la connaissance et de l'acte, qui créent la nature et l'humanité sociale, l'art célèbre, orne, évoque cette réalité préexistante de la connaissance et de l'acte - la nature et l'humanité sociale les enrichit et les complète et, avant tout, crée l'unité concrète, intuitive de ces deux mondes, place l'homme dans la nature, comprise comme son environnement esthétique, humanise la nature et "naturalise" l'homme. ")

On pourrait, même si cela semble d'abord étonnant, appliquer ces considérations aux textes d'enfants. Après tout, le premier récit de rêve :

Une nuit j'ai rêvé d'un monsieur qui venai du plancher. Il venai droit vers ma chambre

il ouvrai le porte il voule me couper ma tête on entendit du bruit puis il m'a couper la tête...

Jean Paul

nous dit peut-être, au delà de la suspension qu'il comporte comme « raconter pour le plaisir », quelque chose de la réalité du rêve (ou d'un rêve symbole du rêve). En même temps il porte des valeurs, une «vision morale »: le texte n'aurait pas de sens si couper la tête n'était pas marqué d'une valeur négative. De la même façon que dans le dialogue de J.-F. Halté avec son fils, le texte est à la fois dans l'espace de suspension, mais en même temps dans la réalité de l'échange entre l'adulte et l'enfant et dans la confrontation des valeurs de l'adulte et de l'enfant comme de l'hétérogénéité de leurs mondes. C'est seulement dans celui des enfants (ou en arrière-fond lointain dans les nôtres ?) que l'existence des 
chiens réels est éclairée par la présence des loups dangereusement fantasmatiques.

Le « littéraire » est, pour reprendre les termes de Bakhtine, « sur le bord du réel ». Si elle peut se maintenir, dans cette position, c'est grâce à son «style ». Ou, selon la formule de Vygotski (Psychologie de l'art, p. 18) : "A nos yeux, l'idée centrale de la psychologie de l'art consiste à reconnaître que la forme artistique l'emporte sur le matériau, ou, ce qui revient au même, que l'art est une technique sociale du sentiment. »C Comme toute formule totalisante, celle-ci est discutable. Elle se précise lorsque Vygotski note que la forme littéraire modifie le thème tel qu'il serait présenté de façon traductible, transparente.

Ce à quoi on peut ajouter au moins deux remarques : une technique est quelque chose de transmissible et de partagé, même s'il y a des «tours de main ». Ici au contraire la technique qu'est le style, c'est ce qui vient à l'auteur plus ou moins consciemment, plus ou moins aisément ou à force de travail, mais c'est « de chacun ». La technique en question est sociale si l'on veut dire par là qu'elle n'est pas naturelle. Mais elle n'est pas sociale comme l'est une institution. Pas plus que n'est institution le rapport instable entre un auteur, une matérialité textuelle et des lecteurs. D'autre part, s'il n'y a pas de perception ou de cognition sans « façon de ressentir », il n’y a pas non plus de «pur ressentir ». D'autant que le moment du ressenti peut rester fascination ou s'ouvrir sur le cours d'autres mouvements de pensée, un peu comme les symboles peuvent aboutir à un figement ou à des ouvertures.

Ce qu'on peut illustrer par un exemple, assurément peu prévisible, tiré de Choses vues de Victor Hugo (1870):

16 juil.

La guerre est déclarée. Cela commence par la Prusse et la France.

Le concile vient de déclarer le pape infaillible.

17 Juillet

Il y a trois jours, le 14 juillet, pendant que je plantais dans mon jardin de Hauteville-House le Chêne des Etats-Unis d'Europe, au même moment, la guerre éclatait en Europe et l'infaillibilité du pape éclatait à Rome.

Dans 100 ans, il n'y aura plus de guerre, il n'y aura plus de pape, et le chêne sera grand.

On peut voir ici, entre autres, le rôle central de la parataxe, de la « mise ensemble » non comme " procédé » mais comme illustrant l'esprit comme lieu de suspension où, contrairement à une vision étriquée de « principe de réalité », on met ensemble ce qui n'était pas destiné à être ensemble. On voit aussi la mise en relation de l'histoire individuelle et de l'histoire globale. Ce texte évoque pour moi le style du Général de Gaulle dans la façon, assurément immodeste, d'identifier son destin et celui du monde. Ce qui ouvre la question de notre mode de réponse : admiration, rire, les deux à la fois ou...

On ajoutera que, certes, il n'y a pas de lien direct entre la vie même d'un individu et son style comme auteur. Reste qu'un enfant ou un quidam qui écriraient comme cela nous inquiéterait...

Mais on pourrait revenir ici à Bakhtine. Ici la littérature est « sur le bord », à la 
fois près et loin du «réel » et de l'éthique. Elle est aussi sur le bord de la «pensée notionnelle » ou de la réflexion.

\subsection{Narratif et notionnel}

Une tradition vieille comme Platon soumet « le récit» au « concept», même si Platon nous rapporte (nous raconte ?) des dialogues (réels ? fictifs ?) et s'il nous raconte des mythes, donc si la question des limites du savoir conceptuel est toujours là. Sans parler de l'ambiguïté de la figure de Socrate, de la difficulté à interpréter le sens du « jeu sérieux » du Parménide ou à déterminer ce que peut signifier vraiment la façon d'interroger l'esclave du Ménon.

Alors ? On peut proposer d'abord que « raconter » est irréductible comme façon de créer du « commun ». Le commun n'est pas l'identique ou le générique. C'est là où le mélange de générique et de particulier qu'est chacun peut être partagé, présent à l'autre comme « symbole », comme figure du commun, même si chacun le réaccentue dans sa lecture.

En ce sens, le récit est « réflexion ». Il n'y a pas là une «fonction » particulière. On ne peut pas séparer ce rapport à la réflexion de ce qui serait une pure nature narrative du récit en tant que tel. C'est aussi une façon d'avoir aux autres des rapports qu'on n'a pas autrement. Ce n'est pas un savoir au sens sérieux. De même qu'il y a là des plaisirs spécifiques, celui de la distance qui devient proche, de la réalité apprivoisée ou encore celui qui provient de ce que le récit nous montre ce qui, en même temps, nous reste opaque. On ne peut dire que Sterne ou Kafka nous manifestent obscurément ou figurativement ce que d'autres devraient ou pourraient nous dire conceptuellement. Il n'y a pas un sur-penseur pour nous dire le vrai du roman.

Autant qu'ils ne se laissent pas enfermer dans l'opposition « général » - «particulier », les récits sont au delà de l'opposition du nécessaire et du contingent. Aussi bien le cours de l'histoire que la vie de chacun comportent du répétitif, des chocs, des rencontres et des modes variés de retentissement. C'est ce que montrent les récits, qui pourraient aussi se présenter comme critique réflexive de la supposée pureté des «idées». Nos idées sont fragiles, soumises aux aléas des situations, des rencontres, à l'esprit du temps, à l'impureté de nos désirs et en premier à celui d'avoir raison. N'est-ce pas cela que nous racontent, entre autres, Dostoïevski ou Tolstoï ? Les «idées », avant d'être vraies ou fausses sont d'abord les idées d'un pauvre homme concret avec la limitation de son horizon, la tendance à se répéter, ses compulsions caractérielles. Et puis elles ne sont que dans leur circulation, avec les aléas que cela comporte. En somme, rappeler tout cela signifie que la pensée romanesque fonctionne comme critique des illusions de survol qui peut régner dans le «monde des idées ». S. Kracauer nous rappelle que les récits des historiens traitent des « avant-dernières choses », non des réalités ultimes, du fond caché. Ce fond caché reste une idée (une illusion ?) à laquelle ni notre pratique ni les récits romanesques n'ont accès. Reste qu'au moins parfois, les récits romanesques nous donnent accès à l'entrelacs, aux surgissements, au hasard, à la diversité des perspectives, à la distance du global et de l'événementiel. Le romancier comme l'historien (quand il a l'art littéraire de raconter) nous présentent les multiples perspectives que nous ne pouvons rencon- 
trer dans la vie effective. L'inachevé qui peut toujours être vu autrement. Mais s'il y a entrelacs, alors il est légitime qu'il y ait plusieurs perspectives, différences entre histoires locales et histoire globale. Et puis, vivons-nous dans le monde des « réalités ultimes »?

\section{Littérature, enfance et transmission}

Quand on traite de l'évolution des enfants on oublie qu'il ne s'agit pas forcément de progrès. Celui-ci est sans doute clair dans la zone des savoir-faire et des savoirs. Dans celui de la façon de s'orienter dans l'espace de suspension, de ressentir, de manifester plus ou moins ce ressenti ou de le « réfléchir », c'est autre chose.

En tout cas, si le « littéraire » c'est ce générique-particulier, sur le bord des autres façons de dire-penser, qui fonctionne en nous donnant à sentir en même temps qu'à penser par sa forme, alors, oui, l'enfant est spontanément littérateur comme le rêveur est « littérateur intime ».

Pour reprendre une distinction de Volochinov, on pourrait dire que la langue de l'école est entre les deux figures qu'il propose de la "langue maternelle » et de la « langue étrangère » : (pp. 107-108) alors que « le mot de la langue maternelle est perçu comme un frère, comme un vêtement familier, mieux encore comme l'atmosphère habituelle dans laquelle nous vivons et nous respirons... », au contraire le mot de la langue étrangère est celui qui «donne à penser » : « dans la conscience historique des peuples, le mot étranger s'est fondu avec l'idée de pouvoir, l'idée de la force, l'idée de sainteté, l'idée de vérité et a obligé la réflexion linguistique à s'orienter de façon privilégiée vers son étude ». Ce qui est créateur en même temps qu'aliénant.

Le « discours de la culture » est toujours un peu celui des « étrangers ». Comme d'ailleurs les " mots de la langue maternelle » ont pu être étranges ou peuvent redevenir tels. Comment alors nous incorporer ce qui, dans sa spécificité, ce qu'il nous apporte comme son style nous est plus ou moins familier plus, ou moins étrange ? Ici aussi on est sur le bord. Le risque est évident : l'étranger peut rester loin, devenir (trop) habituel ou rester quelque chose comme familierétrange.

Alors, assurément, certaines composantes du texte (en partie celles qu'à tort ou raison on a regroupées sous le nom de «langue» : réalisation phonologique ou graphique des unités, syntaxe de base) peuvent ou doivent être partagées. Il est plus difficile de trouver une norme de l'enchaînement textuel. Il n'y a pas des règles universelles de la cohérence, qu'on pourrait se donner ensuite le droit de transgresser. Le récit d'un accident de la circulation du point de vue de la victime qui a été choquée, des bribes sans suite pourront faire sens, dessiner l'allure de la pensée de celui qui sort de l'évanouissement, ce qui n'a pas de raison de comporter un ordre linéaire. Tout comme on peut dire ou écrire «A ce moment, la pluie cessa » sans qu'on ait parlé de pluie au début du texte.

On pourrait proposer que de la narrativité au sens très vague de manifestation de la temporalité peut se retrouver dans différents genres concrets (au sens de pratiques sociales-individuelles variables où tels thèmes sont plus ou moins cor- 
rélés à des modes d'organisation textuels) différents qui ne sont pas que des récits : celui qui avoue sa honte ou les formes variées d'argumentation fondées sur des narrations réelles ou fictives. Mais il est rare qu'on ait justement à faire à cette pure narrativité.

De toute façon, je doute qu'on puisse trouver un ordre de progression pédagogique qui irait universellement du simple au complexe. Ce qui pose la question du rôle de l'enseignant. Relativement facile à saisir (encore que...) lorsqu'il s'agit de faire partager un savoir que les enseignants ont et que les enfants n'ont pas. Mais lorsque un « échec », un «ne pas arriver à dire » est le signe même du mouvement du texte ou lorsque les enfants sont capables de modes du dire ou d'écrire dont l'adulte n'est pas, lui, capable?

De toute façon, chacun d'entre nous est et ne peut être que « particulier-général », et non représentant de la raison universelle. Et lorsque nous « avons raison » contre des plus jeunes, cela ne garantit pas notre statut de « porteurs de la règle ». Certes le maître a, par exemple, des champs de lecture plus larges, il peut suggérer; il n'est pas le détenteur du modèle du bien faire que les autres ne feraient qu'appliquer.

Y a-t-il un enseignement du « littéraire »? Ou fabrique-t-on une espèce particulière de récit : les récits scolaires? Que répondre?

Et, pour finir, qu'en est-il de la « modernité »? Les choses étaient-elles plus simples autrefois? Il y a eu une position « classique » de la culture où la littérature et une certaine littérature étaient au centre et où il allait de soi que cette culture était destinée à l'élite.

D'autre part, elle était avant tout liée à un inventaire limité d'œuvres.

Certes l'élitisme (ou « les élitismes»?) renaissent toujours.

Reste que la figure de « la culture » a beaucoup changé, tant par l'ouverture de la « littérature », que par la culture de masse que prodiguent les media, films, jeux video, musiques transmises...

Que fait ici l'école? Que peut-elle faire ? Ici non plus, je ne me sens pas capable d'une réponse autorisée. Je peux seulement constater comme chacun que la musique et les arts plastiques n'y ont pas une grande place. Pas plus que la pratique des arts visuels du mouvement (ni d'ailleurs de la photographie). Ni non plus la réflexion réfléchie de tout cela.

Certes, il y a le sérieux de « la science » et, surtout, des « affaires ». Ce qu'illustre la question publiquement posée : à quoi peut servir la lecture de la Princesse de Clèves à un futur administrateur ou dirigeant d'entreprise ? Certes pas à sa reconnaissance sociale en tout cas.

Reste à maintenir alors que « la culture » et « la littérature » ne sont pas directement « utiles ».

Après tout, il est bien vrai qu'une majorité d'élèves ne liront plus. Et qu'une toute petite partie écrira autre chose que de l' « utile ». Alors, la « culture » pour quoi faire? On peut toujours affirmer qu'il y a des utilités de l'inutile, qu' on est à l'école pour apprendre et aussi pour « autre chose ». Vouloir formuler une réponse précise a sûrement quelque chose de comique. En assumant ce comique, 
on pourrait, par exemple, proposer que la « littérature », tout comme en général « la culture » sont au centre de ce qui nous manifeste d'autres façons d'être que nos lignes de plus grande pente, sociales et/ou caractérielles, nous aide à ne pas être un homme unidimensionnel, à rechercher non l'identité, mais la capacité à être différent de soi.

A cet égard, l'école peut être ailleurs que dans la modernité. Ce n'est pas forcément un mal.

On peut ajouter qu'ici la pratique précède et domine la théorie. Nous n'avons pas une « théorie vraie » de l'articulation des oraux et des écrits, de ce qui fait qu'un récit (quel que soit son medium) ait un écho en nous, non plus que ce que c'est que le mélange en nous de ce que c'est que le cours de la pensée et de son mixte de langage et de non-langage.

En tout cas, nous rêvons avant de parler. Il y a déjà là un (un peu) différent de nous en nous.

On peut au moins dire que l'école est un lieu où « de l'autre » pourra devenir « de nous ». Et, bien évidemment surtout pour ceux qui n' ont pas « chez eux » ces possibilités d'«altérification ».

Il est bien évident que si le but final est de passer des examens et de faire de bonnes dissertations, il faut une pédagogie frontale. S'il s'agit d'aider, en partie, mais en partie seulement, les élèves à se constituer leurs propres espaces de suspension où gérer, partiellement, ce qui hors d'eux ou en eux, les dépasse, c'est autre chose.

Et puis, autre problème. Après tout la didactique se veut sérieuse et être sérieux n'est-ce pas compter, mesurer? Si j'ai bien compris, une enquête comme Pisa s'occupe davantage de compétences et de cognition que de la relation multiple aux textes, avec ses plaisirs, ses obstacles, ses confusions ses accords et ses violences. Alors, faut-il être « sérieux»?

Et ensuite, que peut devenir l'évocation de l'intime dans l'espace public ? Comment faire pour l'élève qui doit manifester le cours de sa pensée privée dans la lecture publique ? En tout cas, on est dans le domaine du risqué.

Un paradoxe pour finir : on parle toujours de «communication». Mais pour vraiment bien communiquer, en tout cas le « difficile à dire », ne faut-il pas être seul et prendre son temps?

Quant à cet article, on ne croît pas que « la théorie » puisse se développer pour elle-même. Elle est plutôt un moment du dialogue avec ce qui n'est pas elle. C'est ce qui me rassure face à l'évidence de tout ce qui n'a pas été développé ici. Sans parler de la rapidité avec laquelle ce qui a été présenté l'a été. 


\section{Références}

Adorno, T. W. (1985) : «Parataxe» dans Notes sur la Littérature, Paris : Flammarion.

BAKHTINE, M. (1978) : Esthétique et théorie du roman, Paris, N.R.F..

BARTHES, R. (2002) : Le neutre, Cours au Collège de France, (1977-1978), Paris : Le Seuil Imec.

FRANCOIS, F. (2003) : «Unité et multiplicité des temporalités, nos temps et ceux des enfants, ce qui s'en dit, ce qui résiste au discours, ce qui se manifeste à travers le discours », p. 9-33, Qu'est-ce qu'analyser une évolution? Leaple CNRS Paris V.

- (2006) : Rêves, récits de rêves et autres textes. Un essai sur la lecture comme expérience indirecte, Limoges : Lambert-Lucas.

HAlté, J.-F. (1982) : «Apprendre autrement à l'école», dans « Travailler en projet », Pratiques, 36, pp. 5-23.

Hugo, V. (1972) : Choses vues, Paris : Folio classique, 2 vol.

KRACAUER, S. (2006) : L'histoire des avant-dernières choses, Paris : Stock.

KunderA, M. (1986) : L'art du roman, Paris : Gallimard.

PENLOUP, M.-C. (2006) : « Journaux intimes de jeunes enfants. Enjeux didactiques d'une analyse de l'écriture de soi hors école » dans BISHOP, M.-F. et PENLOUP, M.-C. (ed.) «L'écriture de soi et l'école », Repères, 34, pp. 6584.

Proust, M. (1954) : Contre Sainte-Beuve, Paris, Gallimard.

Vygotski, L. S. (2005) : Psychologie de l'art, Paris, La Dispute.

Volochinov, V. N. (1977) : Le marxisme et la philosophie du langage, essai d'application de la méthode sociologique en linguistique, Paris : Minuit.

WinnicotT, D. W. (1991) : «Le bébé en tant que personne », L'enfant et sa famille, Paris : Payot, p. 61. 